\title{
Una visión integral de personas con trayectoria laboral en venta multinivel en Bogotá (Colombia) y Lima (Perú)
}

\author{
Raúl Cabrejos-Burga1, César A. Bernal-Torres ${ }^{2 \star}$, Tamara Pando-Ezcurra1 y Edgar Y. Mayorga ${ }^{3}$ \\ (1) Universidad Alas Peruanas, Escuela de Administración y Negocios Internacionales, Lima, Perú \\ (correo-e: r cabrejos@uap.edu.pe; t pando@uap.edu.pe) \\ (2) Universidad de La Sabana, Escuela Internacional de Ciencias Económicas y Administrativas, Campus Universitario, \\ Chía, Colombia (correo-e: cesar.bernal@unisabana.edu.co) \\ (3) Universidad de La Sabana, Facultad de Ingeniería, Grupo de Investigación Matemáticas y Física Aplicada, Campus \\ Universitario, Chía, Colombia (correo-e: edgar.mayorga@unisabana.edu.co
}

* Autor a quien debe ser dirigida la correspondencia.

Recibido Ago. 6, 2019; Aceptado Oct. 4, 2019; Versión final Oct. 25, 2019, Publicado Abr. 2020

\begin{abstract}
Resumen
Esta investigación analiza los rasgos y las trayectorias laborales de 173 personas que trabajan en venta multinivel. La información se obtuvo mediante encuestas estructuradas a 124 personas en Bogotá (Colombia) y 49 en Lima (Perú). Los resultados de la estadística descriptiva y el análisis de varianza indican que, no existe diferencia significativa entre las personas encuestadas en cuanto a edad, escolaridad y estado civil. Además, la prueba de componentes principales muestra que la principal razón para realizar esta actividad es el deseo de mejorar los ingresos económicos y que los rasgos descriptores más relevantes de estas personas son la autopercepción sobre la calidad de vida y la satisfacción laboral en venta multinivel. Estos resultados aportan evidencia empírica para la comprensión del tema por parte de los académicos y de los responsables de tomar decisiones, en lo referente a la vida laboral y la promoción de empleo.
\end{abstract}

\section{A comprehensive view of people with career paths in multilevel sales in Bogota (Colombia) and Lima (Peru)}

\begin{abstract}
This research analyses the characteristics and career paths of 173 people working in multilevel sales. The information was obtained through structured surveys of 124 people in Bogotá (Colombia) and 49 in Lima (Peru). The results of the descriptive statistics and the analysis of variance indicate that there is no significant difference among the individuals surveyed in terms of age, schooling and marital status. Moreover, the principal component analysis shows that the main reason for carrying out this activity is the desire of improving economic income and that the most relevant descriptive characteristics of these individuals are self-perception about quality of life and job satisfaction in multilevel sales. These results provide empirical evidence for the understanding of the subject by academicians and by decision-makers, regarding working life and employment promotion.
\end{abstract}




\section{INTRODUCCIÓN}

En el contexto de la globalización, asociado a las innovaciones tecnológicas de la información y de la comunicación (TIC), se modifica y reorganiza el trabajo hacía la denominada flexibilización, generando, en las últimas décadas, una profunda transformación sociolaboral (Nam, 2019). Por esto, el crecimiento económico de un sector no se traduce necesariamente en mayores o mejores oportunidades de empleo; al contrario, ante las innovaciones en tecnología y la ardua competencia, el trabajo de las personas se desvaloriza al no ser un factor determinante para el valor de los bienes y los servicios y pierde su referente de primer orden en la esfera laboral (Blanch, 2003).

En este ambiente de globalización se aprecian los siguientes cambios en el orden sociolaboral: descentralización de la producción por la transferencia de las fases productivas a distintos sitios del planeta (Benería, 2006); énfasis en las ventajas competitivas y en los procesos de outsourcing con estructuras más horizontales (Blanch, 2003): diversificación de las modalidades de contrato laboral -trabajos de término definido, de medio tiempo y subcontratación- (De Cuyper et al., 2009). También, por el incremento en la diversidad laboral, desregulación de las condiciones de trabajo, aparición del síndrome de la inseguridad y de la competencia (Benería, 2006); resquebrajamiento del tejido social y de la conciencia colectiva en la sociedad; acentuación en la competencia, las habilidades de aprendizaje y la empleabilidad; y generalización de la percepción de vulnerabilidad, indefensión, exclusión, malestar e insatisfacción de parte de los trabajadores (Olaniyan y Hystad; 2016).

Estos aspectos afectan directamente la vida cotidiana de las personas y, por lo tanto, problematizan las relaciones trabajo-empleo (Blanch, 2003). "Para el trabajador actual, estos cambios le hacen vislumbrar un futuro precario y lleno de riesgos y le llevan a experimentar una sensación permanente de inseguridad, presente incluso en quienes tienen empleo o trabajo formal permanente" (Peralta, 2012; p. 168). Este incremento de la vulnerabilidad se aprecia en una mayor fluidez entre el trabajo remunerado y el no remunerado (especialmente en las mujeres y las personas adultas); gran movilidad entre el empleo informal y el formal y entre el sector público y el privado; marcada variabilidad en los ingresos familiares, aun en las familias que están por encima de la línea de pobreza; y bajos ingresos (Nam, 2019). Esta situación de vulnerabilidad genera gran inseguridad en el presupuesto familiar, inclusive en familias consideradas no pobres, en la medida en que resultan muy frágiles a la hora de cumplir compromisos económicos, especialmente en lo referente a los gastos en educación y vivienda y de mantener un consumo mínimo (Benería, 2006).

A este respecto, el ámbito del trabajo en países como Colombia y Perú se caracteriza por la desigualdad histórica en la distribución de los ingresos, la persistencia de la pobreza y la emigración de grandes cantidades de población, tanto hacia las ciudades como fuera del país; las tendencias de los mercados laborales al incremento de la participación femenina en el trabajo remunerado; la necesidad de las personas sin empleo y, por tanto, marginadas de la cadena productiva, de buscar estrategias de supervivencia y depender del trabajo no monetizado y no incluido en estadísticas oficiales; el flujo constante de la economía, la flexibilización laboral y sus efectos en el desempleo, el subempleo y la informalización; la permanente fluidez entre las actividades dentro y fuera del mercado; y la evolución de la familia en términos demográficos y económicos (Bernal-Torres, et al., 2018; Peralta, 2012 y Roberti, 2012).

Según López-Andreu y Verd (2016) y Roberti (2012), en contextos con dificultades económicas, son las mujeres, los jóvenes y, en general, los desempleados, quienes enfrentan mayores condiciones de precariedad y de segregación, por lo cual se ven necesitados de recurrir a diversas estrategias y prácticas de trabajo. Ante la situación mencionada, una de estas estrategias a la que se recurre para insertarse en el mercado laboral es la venta directa o multinivel, la cual en muchos casos es promocionada por las empresas de este tipo de venta como oportunidad de obtener altos ingresos comercializando sus productos y reclutando más vendedores en sus esquemas (Schiffauer, 2018).

En la actualidad, las trayectorias laborales, entendidas como los rasgos que caracterizan la movilidad laboral de una persona en un período de tiempo, se caracterizan por una mayor movilidad en relación con las formas estables en que hasta finales del siglo XX se desarrollaban las carreras laborales (Roberti, 2012). De acuerdo con Soto (2012), las trayectorias laborales en la actualidad se consideran expresión de nuevos imaginarios que hacen a las personas más disponibles a la flexibilidad y más móviles y la "empresa se concibe como un espacio de transición de intercambio de corto plazo y no como un universo social de pertenencia" (p. 21). A este respecto, según Soto (2012), investigaciones como las realizadas por Grote y Raeder (2009) evidencian que las carreras flexibles tienden a acrecentarse en el mercado de trabajo y particularmente en aquellos sectores más dinámicos y muestran, también, que la opinión de los trabajadores sobre la flexibilidad está lejos del ideal de la autorrealización en el trabajo. 
En virtud de lo antes mencionado, esta investigación se orientó a analizar los rasgos y las trayectorias laborales de las mujeres y los hombres que trabajan en venta directa en la modalidad de multinivel con el fin de identificar los diversos rasgos y recorridos que perfilan la vida laboral de estas personas y, por esto, se orientó a responder los siguientes interrogantes: ¿cuáles son los rasgos y las trayectorias laborales de las personas que trabajan en venta directa multinivel o por catálogo en las ciudades objeto del estudio?

\section{OTROS ANTECEDENTES}

De acuerdo con Guadarrama et al. (2012), cuando se habla de trayectoria laboral se debe ir más allá de una categorización de eventos, en un periodo de tiempo de la vida laboral de una persona, hasta encontrar el sentido otorgado por la propia persona a su situación laboral y las explicaciones que ella misma hace de esta situación a partir de un complejo proceso de mediaciones entre su experiencia individual y el contexto social en el que vive. El estudio de trayectorias, según Roberti (2012), es un medio extraordinario para enmarcar los eventos vitales de las personas en un contexto sociohistórico específico. Para este autor, una manera de abordar estos eventos encuentra como su principal aspecto de análisis los cambios de pautas, prácticas y normas que ocurren en el curso de la vida de las personas, concebidas como instituciones sociales. Y agrega que "los estudios sobre trayectorias generan importantes aportes conceptuales y metodológicos al concebir las articulaciones que existen entre los cambios sociohistóricos y las transformaciones en las subjetividades personales y sociales, como intento de aprehensión de los patrones de cambio de la sociedad en su conjunto a partir del análisis de los cursos de vida individuales" (p. 172).

Para Roberti (2012), la importancia de relacionar el relato que una persona produce sobre su propia vida con un análisis del contexto histórico-social se fundamenta en la existencia de factores objetivos que condicionan los trayectos individuales. Para este autor, lo interesante de las investigaciones con trayectorias es la posibilidad que estas ofrecen para analizar las transformaciones que se han venido presentado en las últimas décadas a escala global, nacional e individual: el surgimiento de la nueva fase trasnacional del capitalismo, las reformas estructurales implementadas, la pérdida de marcos sociales en los que se desarrollaba la vida de una persona y la irrupción de una individualidad sin precedentes. En este sentido, el enfoque de análisis de las trayectorias laborales permite conocer los fenómenos acontecidos al incorporar en su estudio las dimensiones objetivas y subjetivas de la realidad social y laboral de las personas en su quehacer temporal.

Según Orejuela y Correa (2007), los estudios sobre trayectorias laborales han identificado que la movilidad laboral depende de la inversión en capital humano, es decir, que la persona que esté más capacitada o haya alcanzado mayor nivel de cualificación específica y de formación general, tiene mayores posibilidades de permanecer en el mercado de trabajo y de ascender en este ya sea en la misma organización o en otra; que las personas que laboran en empresas sostenibles tienen mejor posibilidad de empleo estable que las personas que están en organizaciones o empresas altamente vulnerables a los cambios del entorno y que muchas veces se ven forzadas a reestructurase o a desaparecer del mercado.

Además, de acuerdo con Castillo et al. (2005), la movilidad del empleo depende del interés de las personas de alcanzar mayores ingresos, del deseo de mayor desarrollo profesional y de satisfacción personal y de la importancia de la armonización entre trabajo y la vida personal; las personas de más edad y de menor nivel educativo son los que cambian menos de ocupación y de industria; es más fácil mantenerse en el mercado laboral para las personas que ya están empleadas que para aquellos que apenas se insertan; la movilidad es mayor entre las mujeres, los más jóvenes, los menos cualificados y los no jefes de hogar y los inmigrantes. A este respecto, estudios recientes sobre trayectorias laborales en España muestran que los adultos jóvenes (hombres y mujeres) son los más afectados por las crisis económicas y, por consiguiente, su vinculación formal con el campo laboral es limitada (Verd et al., 2019; López-Andreu y Verd, 2016).

De acuerdo con Muñiz (2012), en general, los estudios sobre rasgos y trayectorias laborales en América Latina han sido orientados a distintas temáticas tales como migraciones, movilidad social, trabajadores despedidos, asalariados del sector público, movilidad en industrias particulares, trayectorias de microempresarios, trayectorias de género en la economía informal, egresados de determinadas instituciones académicas, etc. Para efectos de este estudio, se analizan los rasgos y las trayectorias de las personas que realizan venta directa o multinivel, un sector en rápido crecimiento en el mundo y en particular en América Latina (WFDSA - World Federation of Direct Selling Associations, 2018), y que ha sido un sector poco estudiado en lo referente a los rasgos y las trayectorias laborales de las personas que trabajan en esta actividad en el contexto latinoamericano.

Para Sousa (2013), en los años 50 del siglo XX, especialmente en América, la venta directa o marketing multinivel ofreció oportunidades para muchos que anteriormente se habían encontrado con barreras debido a la edad, educación o sexo. Pero es solo a partir de los años 90, del mismo siglo XX, cuando se empezó a dar una aproximación con la realidad de lo que es el marketing multinivel de hoy en día (Keed y Vander Nat, 
2013). De acuerdo con los mencionados autores, el marketing multinivel también conocido como marketing en red o marketing referencial es un método de distribución y comercialización de bienes o servicios (casi siempre de consumo masivo) directamente desde un productor o fabricante al consumidor final mediante un sistema de red de personas que buscan generar un ingreso por la referencia de dichos bienes o servicios. Para Kim et al. (2006), el método consiste en que la empresa fabricante incentiva al usuario o consumidor para que refiera sus bienes o servicios a su círculo social y establezca una venta directa.

Para Merlin (2012), este método no depende de la publicidad que las empresas realizan de sus productos o de puntos de venta fijos sino de la referenciación directa que hacen las personas de la red de consumidores. Así, las personas de la red, sobre todo en sus casas, muestran e inducen a otras a probar los productos y a obtener órdenes de pedido. Los productos se suministran, entonces, por la empresa, ya sea directamente al consumidor o a través de la persona de ventas que obtuvo la orden. Según Peterson y Wotruba (1996), el marketing multinivel es un tipo de venta directa cara a cara, fuera de un establecimiento comercial, que no utiliza un sistema común de plan de compensación para sus consumidores o distribuidores, porque la compensación no sólo se hace por las compras o las ventas personales sino también por una participación multinivel por las compras realizadas por las personas referidas o reclutadas por la persona en la red.

Este es un método de ventas donde las personas se vuelven distribuidoras y se ofrece la oportunidad de formar su propia red, así cada nuevo distribuidor tiene la misma oportunidad que otro. Cada distribuidor es considerado una unidad individual responsable del desarrollo de su negocio, teniendo una expectativa proporcional al compromiso adquirido. Es un método en el que cada distribuidor crea su red de distribución basada en un régimen abierto de competencia y responsabilidad, con seguimiento a la cadena que se va formando. La gran diferencia de este método de comercialización con el método tradicional es que crea una red estructurada de venta que, además, es capaz de crear beneficios continuos a lo largo de la vida (Sousa, 2013). De acuerdo con Keed y Vander Nat (2013), en la actualidad, las compañías utilizan variaciones de la estructura de marketing multinivel para vender productos de salud y belleza, artículos de consumo para el hogar, servicios como el transporte e incluso la energía. Son numerosas las empresas de marketing multinivel que reportan ventas anuales de miles de millones de dólares con las redes de distribución en múltiples países.

De acuerdo con Muncy (2012), algunas de las motivaciones de las personas para realizar esta actividad son el acceso al trabajo, la flexibilidad laboral, el desarrollo profesional y la independencia económica. En ese sentido, se suele pensar que las actividades multinivel le ofrecen oportunidades económicas a un grupo considerable de la población dadas sus limitaciones de disponibilidad de tiempo y necesidad de ingresos. En muchos casos, esta actividad es complementaria a la actividad laboral formal pues se combina con otros trabajos remunerados y con el trabajo doméstico no remunerado en la familia (Roberti, 2012). Particularmente las mujeres y, en general, quienes tienen dificultad para acceder al trabajo formal ven en la venta directa una oportunidad de trabajo porque en las empresas que utilizan el multinivel tienen demanda permanente de personal y no hay, aparentemente, exigencias de ingreso respecto a la edad, la escolaridad, el estrato o la capacitación (Sousa, 2013).

En términos de cifras, de acuerdo con la WFDSA - World Federation of Direct Selling Associations (2018), el mercado mundial de venta directa superó los US\$184 billones en ventas y los 108 millones de vendedores. América Latina representó el $13 \%$ del total de las ventas mundiales, contribuyendo con US\$23,4 billones y más de 14 millones de vendedores, fue la región que registró el segundo mayor crecimiento $(5,3 \%)$ del sector entre 2016 y 2017 después de África y Oriente Medio y, de esta región latinoamericana, Colombia y Perú fueron los países en donde más creció la actividad de este sector.

Aunque es innegable el crecimiento de este método en el contexto general de la economía, Legara et al. (2008) cuestionan sus estrategias de comercialización por su similitud con el negocio de las pirámides y por las continuas y amplias expectativas que crea en las personas de la red (especialmente mujeres) de distribuidores y consumidores de obtener altos ingresos con independencia económica y autonomía en la utilización del tiempo, lo cual, muchas veces, más que generar beneficios genera grandes frustraciones. En esta misma dirección, Schiffauer (2018) afirma que, la venta multinivel utiliza estrategias de marketing depredadoras y promesas exageradas, porque los resultados evidencian que solo unos pocos participantes del negocio tienen éxito en su intento de lograr altos ingresos por la realización de esa actividad.

Además, González (2014) agrega que, las empresas multinivel ejercen un control ideológico que violenta la individualidad de los asociados al utilizar un modelo orientado a homogeneizar la forma de pensar y actuar en favor de valores y figuras idealizadas en su negocio. Al respecto, Laczniak y Murphy (2019) advierten que muchos de los sistemas de venta multinivel en los diferentes países del mundo enfrentan problemas legales y éticos y en algunos países este método de comercialización tiene una imagen negativa y poco ética entre muchos consumidores como lo han identificado estudiosos del tema como Cárdenas y Fuchs-Tarlovsky (2018) y Legara et al. (2008). 


\section{METODOLOGÍA}

La información para este estudio se obtuvo de dos grupos de personas (uno de Bogotá - Colombia y otro de Lima - Perú) que laboran en la denominada venta directa en la modalidad multinivel. Para el caso de Bogotá se invitó a participar del estudio a un grupo de 250 personas, de las cuales 198 participaron y, una vez depurada la información, se descartaron 74 encuestas (por respuestas incompletas e información contradictoria), quedando como válidas solo las encuestas de 124 personas (69 mujeres y 55 hombres). Para el caso de Lima se invitó a participar del estudio a un grupo de 150 personas, de las cuales participaron 87 y, con la depuración de la información, se descartaron 38 y quedaron como válidas 49 encuestas (23 de hombres y 26 de mujeres). La muestra se tomó de la forma más aleatoria posible procurando equivalencia entre número de hombres y mujeres. Para esto se acudió a reuniones de participantes de venta directa programadas por diferentes coordinadores de las distintas empresas que realizan esta actividad en las dos ciudades (Bogotá y Lima). El propósito era disponer de una visión integral del sector multinivel en ambas ciudades.

La obtención de la información se hizo mediante encuestas estructuradas realizadas por los investigadores con apoyo de un grupo de estudiantes de los últimos semestres de carrera de la Escuela Internacional de Ciencias Económicas y Administrativas de la Universidad de La Sabana (para el caso de Bogotá) y directamente por los investigadores para el caso de Lima. El formato de la encuesta se diseñó a partir de la revisión de la literatura sobre el tema y en particular con base en los trabajos de Muñiz (2012), Roberty (2012), Orejuela- Gómez y Correa García (2007) y Castillo et al. (2005). La validación previa a la aplicación definitiva de la encuesta se hizo mediante técnica de expertos, para conocer la pertinencia de las preguntas de la encuesta en función de los objetivos y población objeto del estudio. De esa validación se recibieron observaciones referentes a la redacción y al número de preguntas, de tal forma que, de un cuestionario inicial de 25 preguntas se ajustó a 18. Luego se realizó otra validación mediante muestra piloto de 10 personas dedicadas a venta multinivel en Bogotá y a otras 10 en Lima y consecuencia de esto, el cuestionario definitivo se ajustó a 16 preguntas ( 10 abiertas y 6 de opción múltiple).

Realizada la validación del formato de la encuesta, se procedió a establecer contacto directo con las mujeres y los hombres que realizan venta directa para invitarlos a participar del estudio e informales sobre el objetivo y el procedimiento para diligenciar las encuestas. En la encuesta se preguntó sobre datos sociodemográficos de las personas (sexo, edad, estado civil, formación académica, número de hijos, etcétera), datos sobre empleos desempeñados en los últimos diez años (sector económico y tamaño de la empresa, cargo, jornada laboral, tipo de contrato, promedio de ingresos, grado de satisfacción en cada trabajo, capacitación recibida, tiempo de permanencia en la empresa, logros alcanzados, razones para cambio de empleo), datos sobre venta directa (razones para estar en esa actividad, grado de satisfacción en esa actividad, si recomienda o no la venta directa a otras personas) y otra información (logros importantes en toda su actividad laboral, capacidad de ahorro, calidad de vida actual y propósitos, actitud ante la vejez, entre otros aspectos). Para el diligenciamiento de la encuesta se utilizó formato en papel y Word en Tablet y/o computadora y cada una de estas tuvo una duración de 35 minutos.

El procesamiento de la información se realizó con datos agrupados y desagrupados. El análisis de datos agrupados se hizo mediante estadística descriptiva para caracterizar el comportamiento colectivo de las personas de cada ciudad participante del estudio y también para el agregado total de los participantes de las dos ciudades. Esto teniendo en cuenta que el análisis de variables como el sexo, la edad, la escolaridad, el estado civil y el número de hijos de las personas participantes del estudio, con un nivel de confianza del 95 $\%$, indicó que existe similitud de esas variables entre las muestras de las dos ciudades y, por tanto, las muestras se pudieron analizar de forma agrupada. El análisis desagrupado se hizo mediante el análisis de varianza y de componentes principales con el propósito de identificar las variables que tienen el mayor impacto (Blanco-Valbuena, et al., 2018) en la decisión de laborar en la venta multinivel y el grado de satisfacción que se tiene de realizar esta actividad.

Vale señalar que, por el enfoque eminentemente cuantitativo que se dio al procesamiento de la información de las encuestas realizadas, el análisis de la información limita en gran parte la posibilidad de una comprensión más amplia de los relatos expresados por las personas encuestadas en su respuesta a varios de los interrogantes realizados, sin embargo, los resultados numéricos a continuación descritos si reflejan en gran parte la caracterización de las personas dedicadas a la venta multinivel en las ciudades de Bogotá y Lima.

\section{RESULTADOS Y DISCUSIÓN}

Los resultados de este estudio se analizan en tres niveles: en el nivel uno se hace una presentación descriptiva de los rasgos generales de los participantes del estudio y su trayectoria laboral de los últimos 10 años a la fecha de realizar el estudio; en el segundo nivel se hace un estudio inferencial de la independencia y la 
diferencia de medias entre algunas de las variables evaluadas, y, en el tercer nivel, se realiza un análisis por componentes principales buscando asociaciones no evidentes que caractericen las personas que se dedican a la venta directa.

\section{Análisis descriptivo}

La Tabla 1 indica que, del total de personas de Bogotá, 69 son mujeres y 55 son hombres, y del total de personas de Lima, 26 son mujeres y 23 son hombres. También indica que la edad promedio para el conjunto de las personas participantes del estudio es 38,4 años, pero para el caso de las personas de Bogotá dedicadas a estas actividades sus principales rangos de edad son de 30 a 39 años y entre 20 y 29 años, mientras que, para el caso de las personas de Lima, los rangos de edad son entre 20 y 29 años y entre 50 y 59 años. Estos resultados son congruentes con las afirmaciones de Muncy (2012) y Sousa (2013) en lo referente a que la venta multinivel en los últimos años se ha convertido en una opción de trabajo sin discriminación de edad.

Tabla 1: Encuestados según sexo y edad

\begin{tabular}{|l|l|l|l|l|l|l|l|l|l|l|}
\hline \multicolumn{4}{|c|}{ Encuestados por sexo } & \multicolumn{7}{c|}{ Encuestados por edad } \\
\hline & Mujeres & Hombres & Total & $\%$ & {$[20-29]$} & {$[30-39]$} & $40-49]$ & {$[50-59]$} & 59 o más & Prom. \\
\hline Bogotá & 69 & 55 & 124 & 71,2 & $28,2 \%$ & $33,9 \%$ & $21,8 \%$ & $12,1 \%$ & $4,0 \%$ & 37,3 \\
\hline Lima & 26 & 23 & 49 & 28,1 & $30,6 \%$ & $14,3 \%$ & $20,4 \%$ & $30,6 \%$ & $4,1 \%$ & 41,1 \\
\hline Total & 95 & 78 & 173 & & & & & & & 38,4 \\
\hline
\end{tabular}

En cuanto a escolaridad, la mayoría de las personas dedicadas a la venta multinivel manifestaron tener grado profesional (en Bogotá, $56 \%$ y en Lima, $63 \%$ ), seguidas de las que tienen nivel técnico y secundaria. Estos datos se apartan un poco de las afirmaciones de Roberti (2012) quien menciona que la venta multinivel no discrimina el nivel de escolaridad de sus participantes, porque resulta particular el que la mayoría de las personas en Bogotá y Lima son mayoritariamente profesionales. De otra parte, en las dos ciudades, las personas dedicadas a esta actividad son, en su mayoría, solteras (45.1\%) (Tabla 2), lo cual es coherente con las afirmaciones de Orjuela y Correa (2007), quienes afirman que un grupo mayoritario de personas dedicadas a esta actividad son los no jefes de hogar porque son personas que están dispuestas a correr el riesgo de la inestabilidad laboral y el deseo de la independencia económica.

Tabla 2: Encuestados según escolaridad y estado civil

\begin{tabular}{|l|c|c|c|c|c|c|c|c|c|c|}
\hline \multicolumn{9}{|c|}{ Nivel de escolaridad } & \multicolumn{5}{c|}{ Estado civil } \\
\hline & Escuela & Bachiller & Técnico & Profesional & Posgrado & Soltero & Casado & Separado & $\begin{array}{c}\text { Unión } \\
\text { libre }\end{array}$ & Viudo \\
\hline Bogotá & $1,3 \%$ & $19,8 \%$ & $18,8 \%$ & $56,3 \%$ & $3,9 \%$ & $43,5 \%$ & $33,9 \%$ & $9,7 \%$ & $9,7 \%$ & $3,2 \%$ \\
\hline Lima & $1,2 \%$ & $8,5 \%$ & $23,8 \%$ & $63,4 \%$ & $3,0 \%$ & $49,0 \%$ & $42,9 \%$ & $2,0 \%$ & $2,0 \%$ & $4,1 \%$ \\
\hline Total & $1,3 \%$ & $16,4 \%$ & $20,3 \%$ & $58,4 \%$ & $3,6 \%$ & $45,1 \%$ & $35,8 \%$ & $7,5 \%$ & $7,5 \%$ & $3,5 \%$ \\
\hline
\end{tabular}

En general, los resultados de la Tabla 3 indican que quienes se dedican a la venta directa son personas con pocos hijos o ningún hijo. En el caso de Bogotá, el 48,4\% manifestaron tener entre 1 y 2 hijos y el 37,9\% ningún hijo, y en Lima el 40,8 \% ningún hijo y el 36,7\% entre 1 y 2 hijos. Datos que indican que, la venta directa en las ciudades estudiadas es poco atractiva para las personas que tienen varios hijos, lo cual puede obedecer a la inestabilidad laboral que genera esta actividad, a la demanda de horarios adicionales de trabajo o a la incertidumbre de obtener altos ingresos con independencia económica y autonomía en la utilización del tiempo, por parte de quienes se dedican a este tipo de venta como afirman Legara et al. (2008). Los datos de esta tabla también muestran que, las personas dedicadas a la venta directa tienen vivienda propia (en Bogotá, el $59,7 \%$ y en Lima el $42,9 \%$ ), indicando que cuentan con cierto grado de estabilidad económica.

Tabla 3: Encuestados según número de hijos y posesión de vivienda

\begin{tabular}{|c|c|c|c|c|c|c|c|}
\hline \multicolumn{6}{|c|}{ Número de hijos } & \multicolumn{2}{|c|}{ Posesión de vivienda } \\
\hline & 0 & 102 & $3 \circ 4$ & Más de 4 & Promedio & Sí & No \\
\hline Bogotá & $37,9 \%$ & $48,4 \%$ & $12,1 \%$ & $1,6 \%$ & 1,28 & $59,7 \%$ & $40,3 \%$ \\
\hline Lima & $40,8 \%$ & $36,7 \%$ & $20,4 \%$ & $2,0 \%$ & 1,32 & $42,9 \%$ & $57,1 \%$ \\
\hline
\end{tabular}

En cuanto a su historia laboral de los últimos diez años, en Bogotá, la mayoría de los participantes (54\%) manifestó haber tenido tres empleos, mientras que en Lima la mayoría señaló haber tenido solo uno (55,1\%) (Tabla 4). Esto puede indicar que las personas en Lima tienen más estabilidad laboral y posibilidades de 
ascenso que las de Bogotá o que en esta ciudad son más propensos a cambiar de empleo en busca de nuevas oportunidades o con el propósito de mejorar las condiciones laborales (Orjuela y Correa, 2007). Igualmente, los datos de la Tabla 4 indican que, los principales motivos de ingreso al primer trabajo de los entrevistados fue la necesidad de trabajar (Bogotá, $48 \%$ y Lima, 46,9\%) seguido de la disponibilidad de contar con recomendaciones (Bogotá, 31,5\% y Lima, 20,4\%), lo cual concuerda con las razones de acceso al trabajo por parte de la mayoría de las personas en las economías emergentes y distinto en las economías desarrolladas donde la razón de trabajar es la identificación de oportunidades de trabajo y desarrollo laboral y personal (Cepal - IOT, 2018).

Tabla 4: Número de empleos diferentes a multinivel y motivo de ingreso al primer empleo

\begin{tabular}{|l|c|c|c|c|c|c|c|c|}
\hline \multicolumn{4}{|c}{ Número de empleos } & \multicolumn{4}{c|}{ Motivo de ingreso al primer empleo } \\
\hline & Ninguno & Uno & Dos & $\begin{array}{c}\text { Tres o } \\
\text { más }\end{array}$ & Interés & Necesidad & Oportunidad & Recomendación \\
\hline Bogotá & $0,8 \%$ & $15,3 \%$ & $29,8 \%$ & $54,0 \%$ & $4,0 \%$ & $48,4 \%$ & $16,1 \%$ & $31,5 \%$ \\
\hline Lima & $6,1 \%$ & $55,1 \%$ & $34,7 \%$ & $4,1 \%$ & $20,4 \%$ & $46,9 \%$ & $12,2 \%$ & $20,4 \%$ \\
\hline
\end{tabular}

Para el caso de las personas que manifestaron tener un solo empleo durante los últimos diez años y que son el 26,6\% del conjunto de los participantes del estudio, en general, estas trabajan en porcentajes similares en las empresas de los diferentes tamaños (desde micro hasta las grandes); el $40 \%$ de ellas manifestaron tener contrato a término indefinido, el $31.1 \%$ otro tipo de contrato y el $28.9 \%$ contrato a término fijo. El $60 \%$ de ellas trabajar en cargos con funciones operativas, no haber tenido acceso a capacitación para el desempeño de sus actividades $(62,2 \%$ ) y estar satisfechas con el salario recibido versus el esfuerzo realizado en su trabajo $(53,3 \%)$ (Tabla 5). Esto confirma que los rasgos de la nueva economía caracterizada por la diversificación de las modalidades de contrato laboral (De Cuyper et al., 2009) son hoy una realidad en el mundo laboral de las personas en las ciudades estudiadas.

Tabla 5: Personas que sólo registraron un empleo diferente a multinivel en los últimos 10 años

\begin{tabular}{|l|c|l|c|l|c|l|c|c|c|}
\hline $\begin{array}{c}\text { Tamaño de la empresa } \\
\text { donde labora }\end{array}$ & \multicolumn{3}{|c|}{ Tipo de contrato laboral } & \multicolumn{2}{c|}{ Nivel del cargo } & \multicolumn{3}{c|}{$\begin{array}{c}\text { Acceso a } \\
\text { capacitación }\end{array}$} & \multicolumn{2}{c|}{$\begin{array}{c}\text { Satisfacción ingreso } \\
\text { vs. trabajo }\end{array}$} \\
\hline Micro & $24,4 \%$ & Fijo & $28,9 \%$ & Operativo & $60,0 \%$ & No & $62,2 \%$ & \multicolumn{2}{|c|}{} \\
\hline Pequeña & $24,4 \%$ & Indefinido & $40,0 \%$ & Técnico & $20,0 \%$ & Sí & $37,8 \%$ & No & $46,7 \%$ \\
\hline Mediana & $24,4 \%$ & Otro & $31,1 \%$ & Profesional & $15,6 \%$ & & & Sí & $53,3 \%$ \\
\hline Grande & $26,7 \%$ & & & Directivo & $4,4 \%$ & & & & \\
\hline
\end{tabular}

Los datos de la Tabla 6 indican que el $37,8 \%$ de las personas que en los últimos diez años han tenido un solo empleo, manifestaron tener jornada de trabajo de entre 9 y 12 horas diarias; el $55,6 \%$ que reciben salario por debajo de 300 dólares mensuales y 35,6 \% entre 300 y 600 dólares; el 46,7 \% dice tener percepción positiva del jefe y que su principal logro fue adquirir experiencia (40\%), lo cual indica que una razón por la cual estas personas decidieron realizar venta directa es la de mejorar sus ingresos y adquirir experiencia laboral como lo afirman Orjuela y Correa (2007).

Tabla 6: Rasgos del empleo de los encuestados que registraron un empleo diferente a multinivel

\begin{tabular}{|l|c|l|c|l|c|l|c|}
\hline \multicolumn{2}{|c|}{ Horas de trabajo diario } & \multicolumn{2}{|c|}{ Salario (USD) } & \multicolumn{2}{c|}{ Percepción del jefe } & \multicolumn{2}{c|}{ Principal logro } \\
\hline 4 o menos & $11,1 \%$ & 300 o menos & $55,6 \%$ & Pésima & $2,2 \%$ & Ascenso laboral & $6,7 \%$ \\
\hline 5 a 8 & $31,1 \%$ & 301 a 600 & $35,6 \%$ & Mala & $15,6 \%$ & Éxito laboral & $17,8 \%$ \\
\hline 9 a 12 & $37,8 \%$ & 601 a 1300 & $8,9 \%$ & Regular & $26,7 \%$ & Independización & $2,2 \%$ \\
\hline Más de 12 & $4,4 \%$ & 1301 a 2000 & $0,0 \%$ & Buena & $46,7 \%$ & Mejores ingresos & $11,1 \%$ \\
\hline Flexible & $15,60 \%$ & 2001 o más & $0,0 \%$ & Excelente & $6,7 \%$ & Ninguno & $22,2 \%$ \\
\hline & & & & Sin jefe & $2,2 \%$ & $\begin{array}{l}\text { Adquirir } \\
\text { experiencia }\end{array}$ & $40,0 \%$ \\
\hline
\end{tabular}

Los datos de la Tabla 7 indican que, para el caso de las personas que registraron haber tenido dos empleos diferentes a venta directa en los últimos 10 años y que representan el 31,2\% del conjunto de las participantes del estudio, el 37,9\% de ellas manifestaron trabajar en empresas grandes en el segundo empleo, porcentaje 
que fue menor en el primer empleo. El porcentaje de las personas que tuvieron contrato a término indefinido en el primer empleo (31,5\%) aumentaron porcentualmente en el segundo empleo (38,7 \%), lo cual puede indicar que las personas tienden a buscar estabilidad laboral. El porcentaje de las personas que en su primer empleo (E1) desempeñaron cargo en el nivel profesional (12\%) se incrementó al 26,6 \% en el segundo empleo (E2). En cuanto a capacitación, también se presentó mejora para el segundo empleo al pasar del 49 $\%$ al $53,2 \%$.

Tabla 7: Personas que registraron dos empleos diferentes a multinivel en los últimos 10 años

\begin{tabular}{|c|c|c|c|c|c|c|c|c|c|c|c|}
\hline \multicolumn{3}{|c|}{ Tamaño de la empresa } & \multicolumn{3}{|c|}{ Tipo de contrato } & \multicolumn{3}{|c|}{ Nivel del cargo } & \multicolumn{3}{|c|}{ Capacitación } \\
\hline & $\mathrm{E} 1$ & E2 & & $\mathrm{E} 1$ & E2 & & $\mathrm{E} 1$ & E2 & & $\mathrm{E} 1$ & E2 \\
\hline Micro & $21,0 \%$ & $18,5 \%$ & Fijo & $33,1 \%$ & $28,2 \%$ & Operativo & $79,0 \%$ & $44,4 \%$ & Sí & $49,2 \%$ & $53,2 \%$ \\
\hline Pequeña & $19,4 \%$ & $18,5 \%$ & Indefinido & $31,5 \%$ & $38,7 \%$ & Técnico & $4,0 \%$ & $19,4 \%$ & No & $50,8 \%$ & $46,8 \%$ \\
\hline Mediana & $32,3 \%$ & $25,0 \%$ & Otro & $35,5 \%$ & $33,1 \%$ & Profesional & $12,1 \%$ & $26,6 \%$ & & & \\
\hline Grande & $27,4 \%$ & $37,9 \%$ & & & & Directivo & $4,8 \%$ & $9,7 \%$ & & & \\
\hline
\end{tabular}

La satisfacción por el salario en el segundo empleo (46 \% entre 300 y 600 dólares y $20 \%$ entre 601 y 1300 dólares) mejoró porcentualmente del primero (70,2 \%) al segundo empleo (75 \%) pero, aunque un porcentaje de personas disminuyó su extensa jornada de trabajo (9 a 12 horas, 54 \% de las personas), el porcentaje con estos horarios siguió siendo alto en el segundo empleo $(49,2 \%)$ y la satisfacción con los jefes se redujo del primero $(62,1 \%)$ al segundo empleo (51,6 \%) (Tabla 8$)$.

Tabla 8: Rasgos de personas con dos empleos diferentes a multinivel en los últimos 10 años

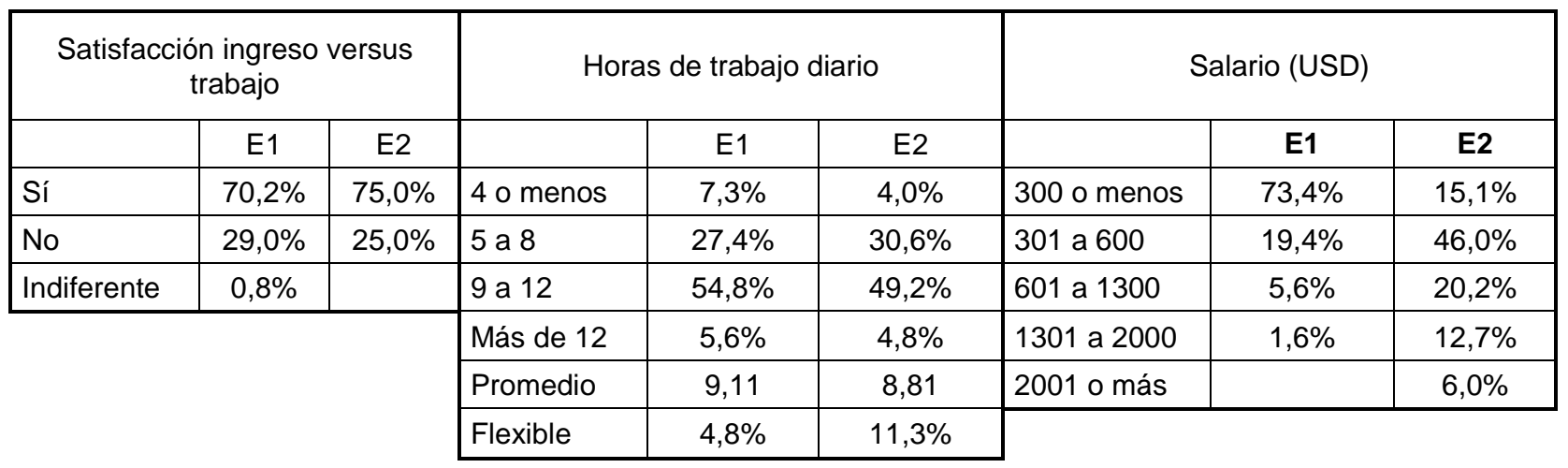

Los datos de la Tabla 9 muestran que, las personas que tuvieron dos empleos diferentes a la venta directa, en general, en el segundo empleo disminuyó el porcentaje de personas que manifestaron tener buenas relaciones con sus jefes, al pasar del $62 \%$ en el primer empleo al 51,6\% en el segundo empleo. Esto indica que en el segundo empleo las personas sintieron tener relaciones con los jefes menos favorables que las establecidas en el primer empleo. Aunque, similar a las personas con un solo empleo diferente a la venta directa, su principal logro en el trabajo fue ganar experiencia $(40,3 \%)$ y el éxito laboral $(13,7)$, sin embargo, estos porcentajes disminuyeron al pasar del primero al segundo empleo.

Tabla 9: Relación con el jefe y principal logro en el trabajo en personas con dos empleos

\begin{tabular}{|l|c|c|l|c|c|}
\hline \multicolumn{3}{|c|}{ Relación con el jefe } & \multicolumn{3}{c|}{ Principal logro } \\
\hline & E1 & E2 & & E1 & E2 \\
\hline Pésima & $0,8 \%$ & $2,4 \%$ & Ascenso laboral & $8,9 \%$ & $10,5 \%$ \\
\hline Mala & $4,8 \%$ & $4,0 \%$ & Éxito laboral & $13,7 \%$ & $13,7 \%$ \\
\hline Regular & $15,3 \%$ & $6,5 \%$ & Independización & $4,8 \%$ & $6,5 \%$ \\
\hline Buena & $62,1 \%$ & $51,6 \%$ & Mejores ingresos & $5,6 \%$ & $14,5 \%$ \\
\hline Excelente & $15,3 \%$ & $13,7 \%$ & Ninguno & $13,7 \%$ & $14,5 \%$ \\
\hline Sin jefe & $1,6 \%$ & $21,8 \%$ & Ganar Experiencia & $53,2 \%$ & $40,3 \%$ \\
\hline
\end{tabular}

Para el caso de las personas que registraron haber tenido tres empleos diferentes a venta directa en los últimos diez años (39,9\% del conjunto de las participantes del estudio), se observa incremento en el 
porcentaje de las personas que laboran en empresas grandes, mientras que el porcentaje de las que trabajan en microempresas tiende a ser estable (24\%). De otra parte, en su tercer empleo, el 50,7\% de las personas manifestaron tener contrato laboral a término indefinido y, en su mayoría, desempeñarse en cargos operativos $(53,6 \%)$ y en cargos profesionales $(30,4 \%)$ (Tabla 10$)$.

Tabla 10: Personas que registraron tres empleos diferentes a multinivel en los últimos 10 años

\begin{tabular}{|c|c|c|c|c|c|c|c|c|c|c|c|}
\hline \multicolumn{4}{|c|}{ Tamaño de la empresa } & \multicolumn{4}{|c|}{ Tipo de contrato } & \multicolumn{4}{|c|}{ Nivel del cargo } \\
\hline & E1 & E2 & E3 & & $\mathrm{E} 1$ & E2 & E3 & & $\mathrm{E} 1$ & E2 & E3 \\
\hline Micro & $23,2 \%$ & $23,2 \%$ & $24,6 \%$ & Fijo & $39,1 \%$ & $31,9 \%$ & $21,7 \%$ & Operativo & $82,6 \%$ & $53,6 \%$ & $53,6 \%$ \\
\hline Pequeña & $20,3 \%$ & $23,2 \%$ & $15,9 \%$ & Indefinido & $31,9 \%$ & $37,7 \%$ & $50,7 \%$ & Técnico & $4,3 \%$ & $13,0 \%$ & $8,7 \%$ \\
\hline Mediana & $29,0 \%$ & $23,2 \%$ & $20,3 \%$ & Otro & $29,0 \%$ & $30,4 \%$ & $27,5 \%$ & Profesional & $8,7 \%$ & $26,1 \%$ & $30,4 \%$ \\
\hline Grande & $27,5 \%$ & $30,4 \%$ & $39,1 \%$ & & & & & Directivo & $4,3 \%$ & $7,2 \%$ & $7,2 \%$ \\
\hline
\end{tabular}

Los datos de la Tabla 11 indican que, en general, tiende a haber similitud en las personas que reciben y las que no reciben capacitación en sus diferentes empleos; el porcentaje de las personas que manifestaron estar satisfechas con su salario recibido durante todos los empleos es superior al $73 \%$ y un porcentaje superior al $60 \%$ dice tener jornadas de trabajo entre 9 y 12 horas diarias; mientras que, solo un porcentaje inferior al $20 \%$ afirman trabajar menos de 8 horas diarias. Estos datos indican, que en general, más del $60 \%$ de las personas encuestadas dedican más de 9 horas diarias al trabajo.

Tabla 11: Capacitación, satisfacción y horas de trabajo diario en personas con tres empleos diferentes a multinivel en los últimos 10 años

\begin{tabular}{|l|c|c|c|c|c|c|c|c|c|c|c|}
\hline \multicolumn{4}{|c|}{ Capacitación } & \multicolumn{3}{c|}{ Satisfacción ingreso vs trabajo } & \multicolumn{5}{c|}{ Horas de trabajo diario } \\
\hline & E1 & E2 & E3 & & E1 & E2 & E2 & & E1 & E2 & E3 \\
\hline No & $43,5 \%$ & $53,6 \%$ & $46,4 \%$ & No & $23,2 \%$ & $26,1 \%$ & $18,8 \%$ & 4 o menos & $7,2 \%$ & $2,9 \%$ & $2,9 \%$ \\
\hline Sí & $56,5 \%$ & $46,4 \%$ & $53,6 \%$ & Sí & $76,8 \%$ & $73,9 \%$ & $81,2 \%$ & 5 a 8 & $21,7 \%$ & $24,6 \%$ & $\begin{array}{c}20,3 \\
\%\end{array}$ \\
\hline
\end{tabular}

La Tabla 12, muestra que, en general, las personas mejoraron el salario al pasar de un empleo al siguiente, pero el mayor porcentaje $(44,9 \%)$ de las personas con tercer empleo diferente al de venta directa ganan en promedio entre 300 y 600 dólares americanos. También, la tabla indica que las personas manifiestan tener buena (58\%) y excelente (18\%) percepción del jefe y que su principal logro es el haber recibido capacitación por parte de las empresas.

Tabla 12: Salario promedio, percepción de la relación con el jefe y principal logro de las personas con tres trabajos diferentes a multinivel en los últimos 10 años

\begin{tabular}{|l|c|c|c|c|c|c|c|l|c|c|c|}
\hline \multicolumn{5}{|c|}{ Salario (USD) } & \multicolumn{4}{c|}{ Percepción del jefe } & \multicolumn{4}{c|}{ Principal logro } \\
\hline & E1 & E2 & E3 & & E1 & E2 & E3 & & E1 & E2 & E3 \\
\hline $\begin{array}{l}300 \text { o } \\
\text { menos }\end{array}$ & $71,0 \%$ & $42,0 \%$ & $26,1 \%$ & Pésima & $1,4 \%$ & $2,9 \%$ & $0,0 \%$ & Ascenso laboral & $7,2 \%$ & $10,1 \%$ & $5,8 \%$ \\
\hline 301 a 600 & $24,6 \%$ & $43,5 \%$ & $44,9 \%$ & Mala & $4,3 \%$ & $5,8 \%$ & $2,9 \%$ & Éxito laboral & $18,8 \%$ & $15,9 \%$ & $15,9 \%$ \\
\hline 601 a 1300 & $2,9 \%$ & $8,7 \%$ & $14,5 \%$ & Regular & $10,1 \%$ & $7,2 \%$ & $10,1 \%$ & Independización & $4,3 \%$ & $7,2 \%$ & $7,2 \%$ \\
\hline $\begin{array}{l}1301 \text { a } \\
2000\end{array}$ & $1,4 \%$ & $4,3 \%$ & $8,7 \%$ & Buena & $63,8 \%$ & $56,5 \%$ & $58,0 \%$ & $\begin{array}{l}\text { Mejores } \\
\text { ingresos }\end{array}$ & $1,4 \%$ & $7,2 \%$ & $10,1 \%$ \\
\hline 2001 o más & & $1,4 \%$ & $5,8 \%$ & Excelente & $18,8 \%$ & $18,8 \%$ & $18,8 \%$ & Ninguno & $10,1 \%$ & $18,8 \%$ & $14,5 \%$ \\
\hline
\end{tabular}


De otra parte, los datos de la Tabla 13 indican que, del total de personas encuestadas en Bogotá, el 37,1 \% de ellas, en algún momento durante los últimos diez años, enfrentaron el problema del desempleo, mientras que en Lima las personas que enfrentaron desempleo fueron solo el 10,2 \%. Estos datos indican que, en el periodo de análisis, las personas en Bogotá - Colombia tuvieron menores oportunidades de empleo debido en gran parte a la falta de demanda de trabajadores por parte del sector productivo, a la reducción del crecimiento del producto interno bruto, a los impactos de las reformas tributarias de últimos años, a la caída de los precios del petróleo, a la política contraccionista de reducción del gasto público, a la fuerte polarización política y la incertidumbre que esta genera sobre las decisiones económicas y a la llegada masiva de venezolanos al país.

Tabla 13: Personas que realizan venta multinivel y afrontaron desempleo en los últimos 10 años

\begin{tabular}{|c|c|c|c|}
\hline & Sí & No & Total \\
\hline Lima & $5(10,2 \%)$ & $44(89,8 \%)$ & 49 \\
\hline Bogotá & $46(37,1 \%)$ & $78(62,9 \%)$ & 124 \\
\hline Total & 51 & 122 & 173 \\
\hline
\end{tabular}

Del total de personas que afrontaron la situación de desempleo, el 41,2 \% estuvieron desempleadas menos de un año y el 37,3 \% entre 1 y 2 años. Las principales razones del desempleo fueron la falta de oferta laboral $(43,1 \%)$ y las situaciones personales $(33,3 \%)$. Las actividades más desempeñadas por estas personas durante el desempleo fueron el trabajo independiente $(19,6 \%)$ y el estudio $(11,8 \%)$ y la forma como se financiaron durante ese tiempo fue su ingreso por concepto del trabajo independiente $(58,8 \%)$ y la venta directa $(23,5 \%)$ (Tabla 14)

Tabla 14: Duración, razón, actividad realizada y fuente de financiación del periodo de desempleo

\begin{tabular}{|c|c|c|c|c|c|c|c|}
\hline \multicolumn{2}{|c|}{$\begin{array}{l}\text { Periodo más largo sin } \\
\text { empleo formal (Años) }\end{array}$} & \multicolumn{2}{|c|}{$\begin{array}{l}\text { Razón del periodo más largo } \\
\text { sin empleo formal }\end{array}$} & \multicolumn{2}{|c|}{$\begin{array}{l}\text { Actividad en periodo sin } \\
\text { empleo formal }\end{array}$} & \multicolumn{2}{|c|}{$\begin{array}{l}\text { Financiación en periodo sin } \\
\text { empleo formal }\end{array}$} \\
\hline $\begin{array}{c}\text { Menos de } \\
1 \\
\end{array}$ & $41,2 \%$ & Estudio & $13,7 \%$ & $\begin{array}{l}\text { Asuntos } \\
\text { personales }\end{array}$ & $5,9 \%$ & $\begin{array}{c}\text { Actividades } \\
\text { independientes }\end{array}$ & $5,9 \%$ \\
\hline 1 a 2 & $37,3 \%$ & $\begin{array}{l}\text { Proyecto de } \\
\text { independización }\end{array}$ & $9,8 \%$ & Estudiar & $11,8 \%$ & Ahorros & $11,8 \%$ \\
\hline 2 a 5 & $17,6 \%$ & $\begin{array}{l}\text { Razones } \\
\text { personales }\end{array}$ & $33,3 \%$ & $\begin{array}{l}\text { Trabajo en el } \\
\text { hogar }\end{array}$ & $7,8 \%$ & $\begin{array}{c}\text { Trabajos } \\
\text { independientes }\end{array}$ & $58,8 \%$ \\
\hline Más de 5 & $3,9 \%$ & $\begin{array}{l}\text { Sin ofertas } \\
\text { laborales }\end{array}$ & $43,1 \%$ & $\begin{array}{l}\text { Trabajos } \\
\text { independientes }\end{array}$ & $19,6 \%$ & Venta directa & $23,5 \%$ \\
\hline \multirow[t]{2}{*}{ (2) } & & & & Venta directa & $25,5 \%$ & & \\
\hline & & & & Ninguna & $29,4 \%$ & & \\
\hline
\end{tabular}

Ahora, en lo referente específicamente a la venta directa, en general, las personas participantes del estudio están en esta actividad en los rangos de dos a cinco años (43,9\%) y de más de 5 años (28,9\%), lo cual indica que la venta directa es una actividad en la que las personas tienden a permanecer vinculadas por varios años. También, en general, las personas entrevistadas en las dos ciudades y que se dedican a esta actividad manifiestan estar satisfechas (el 64,2 \%) y muy satisfechas (24,9\%) (Tabla 15), indicando con esto que, esta actividad genera satisfacción para quienes se dedican a esa labor.

Tabla 15: Tiempo en venta multinivel y satisfacción en esa actividad

\begin{tabular}{|l|c|c|c|c|c|c|c|c|c|}
\hline \multicolumn{5}{|c|}{ Tiempo en venta multinivel } & \multicolumn{4}{c|}{ Satisfacción en la actividad } \\
\hline & $\begin{array}{c}\text { Menos } \\
\text { de 1 }\end{array}$ & 1 a 2 & 2 a 5 & $\begin{array}{c}\text { Más de } \\
5\end{array}$ & Promedio & Insatisfecho & $\begin{array}{c}\text { Levemente } \\
\text { satisfecho }\end{array}$ & Satisfecho & $\begin{array}{c}\text { Muy } \\
\text { satisfecho }\end{array}$ \\
\hline Bogotá & $4,8 \%$ & $24,2 \%$ & $43,5 \%$ & $27,4 \%$ & 5,03 & $5,6 \%$ & $7,3 \%$ & $66,1 \%$ & $21,0 \%$ \\
\hline Lima & $2,0 \%$ & $20,4 \%$ & $44,9 \%$ & $32,7 \%$ & 4,96 & $2,0 \%$ & $4,1 \%$ & $59,2 \%$ & $34,7 \%$ \\
Total & $4,0 \%$ & $23,1 \%$ & $43,9 \%$ & $28,9 \%$ & 5,01 & $4,6 \%$ & $6,4 \%$ & $64,2 \%$ & $24,9 \%$ \\
\hline
\end{tabular}

Las principales razones por las que las personas participantes del estudio realizan la venta directa son los beneficios económicos que ofrece esta actividad $(60,1 \%)$ y la flexibilidad para el uso del tiempo (15 \%) y en menos proporción el considerar a esta actividad como una oportunidad exclusiva de trabajo (6.2\%); sin embargo, un porcentaje alto de personas (90\%) si recomienda esta actividad como un complemento a una actividad laboral principal (Tabla 16). 
Tabla 16: Razón de laborar en multinivel y recomendación de esa labor

\begin{tabular}{|c|c|c|c|c|c|c|c|}
\hline \multicolumn{6}{|c|}{ Razón de laborar en venta directa } & \multicolumn{2}{|c|}{ Recomienda } \\
\hline & $\begin{array}{c}\text { Beneficios } \\
\text { económicos }\end{array}$ & $\begin{array}{c}\text { Flexibilidad } \\
\text { del tiempo }\end{array}$ & $\begin{array}{c}\text { Interés de } \\
\text { servicio }\end{array}$ & Oportunidad & $\begin{array}{l}\text { Beneficios de } \\
\text { los productos }\end{array}$ & Sí & No \\
\hline Bogotá & $64,5 \%$ & $16,1 \%$ & $8,9 \%$ & $6,5 \%$ & $4,0 \%$ & $87,1 \%$ & $12,9 \%$ \\
\hline Lima & $49,0 \%$ & $12,2 \%$ & $16,3 \%$ & $6,1 \%$ & $16,3 \%$ & $93,9 \%$ & $6,1 \%$ \\
\hline Total & $60,1 \%$ & $15,0 \%$ & $11,0 \%$ & $6,4 \%$ & $7,5 \%$ & $89,0 \%$ & $11,0 \%$ \\
\hline
\end{tabular}

Las principales razones por las que estas personas recomendarían la venta directa a otras personas es la mejora de los ingresos que genera esta actividad $(40,5 \%)$ y la independencia de los jefes y la flexibilidad con que se puede aprovechar el tiempo (34,1\%) y la razón por la que menos recomendaría la venta directa 0 multinivel es porque esa actividad demanda mucho esfuerzo (Tabla 17).

Tabla 17: Razones por las que recomendaría $(\mathrm{S} / \mathrm{N})$ venta multinivel

\begin{tabular}{|l|c|c|c|c|c|}
\hline & $\begin{array}{c}\text { Mejores } \\
\text { ingresos }\end{array}$ & $\begin{array}{c}\text { Independencia / } \\
\text { manejo del tiempo }\end{array}$ & $\begin{array}{c}\text { Beneficios de los } \\
\text { productos }\end{array}$ & $\begin{array}{c}\text { Relación con } \\
\text { otras personas }\end{array}$ & $\begin{array}{c}\text { Demanda } \\
\text { esfuerzo }\end{array}$ \\
\hline Bogotá & $41,9 \%$ & $35,5 \%$ & $6,5 \%$ & $3,2 \%$ & $10,5 \%$ \\
\hline Lima & $36,7 \%$ & $30,6 \%$ & $12,2 \%$ & $14,3 \%$ & $4,1 \%$ \\
\hline Total & $40,5 \%$ & $34,1 \%$ & $8,1 \%$ & $6,4 \%$ & $8,7 \%$ \\
\hline
\end{tabular}

Los datos de la Tabla 18, muestran que, al comparar la venta directa con sus otros trabajos por parte de los participantes del estudio, para el caso de las personas en Bogotá su mayor satisfacción laboral ha sido la de otros trabajos $(53,2 \%)$ más que la venta directa, mientras que para el caso de las personas de la ciudad de Lima la mayor satisfacción de su vida laboral ha sido la venta directa $(73,5 \%)$ más que sus otros trabajos. Esta mayor satisfacción con la venta directa respecto de otros trabajos por parte de las personas de Lima, frente a sus homólogos en Bogotá, puede obedecer a que las personas en Lima perciben la labor de venta directa como altamente positiva para su familia (Tabla 18) y como una gran fuente para su mejora económica (Tabla 20).

También, los datos de la Tabla 18, muestran que, el conjunto de las personas encuestadas manifiesta tener una calidad de vida actual buena $(65,9 \%)$ y excelente (19.1\%), lo cual no indica que, la actividad en la venta directa sea la razón del bienestar de estas personas, porque esta información se obtuvo de la respuesta a una pregunta referida a calidad de vida en sentido genérico.

Tabla 18: Empleo de mayor satisfacción y calidad de vida actual

\begin{tabular}{|c|c|c|c|c|c|c|c|c|}
\hline \multicolumn{3}{|c|}{ Empleo de mayor satisfacción } & \multicolumn{4}{c|}{ Calidad de vida actual } \\
\hline & $\begin{array}{c}\text { Venta } \\
\text { directa }\end{array}$ & $\begin{array}{c}\text { Venta directa } \\
\text { y otro }\end{array}$ & Otro & Mala & Regular & Normal & Buena & Excelente \\
\hline Bogotá & $42,7 \%$ & $4,0 \%$ & $53,2 \%$ & $0,8 \%$ & $2,4 \%$ & $8,9 \%$ & $70,2 \%$ & $17,7 \%$ \\
\hline Lima & $73,5 \%$ & $2,0 \%$ & $24,5 \%$ & $0,0 \%$ & $8,2 \%$ & $14,3 \%$ & $55,1 \%$ & $22,4 \%$ \\
\hline Total & $51,4 \%$ & $3,5 \%$ & $45,1 \%$ & $0,6 \%$ & $4,0 \%$ & $10,4 \%$ & $65,9 \%$ & $19,1 \%$ \\
\hline
\end{tabular}

De otra parte, los datos de la Tabla 19 indican que, la gran mayoría $(84,4 \%)$, de las personas encuestadas perciben que la venta multinivel ha afectado de forma positiva su vida familiar, mientras que, solo el $7 \%$ percibe que los ha afectado de forma negativa y, además, que esta actividad les permite disponer de capacidad de ahorro $(74,6 \%)$.

Tabla 19: Implicación de la venta multinivel en la vida familiar y en la capacidad de ahorro

\begin{tabular}{|l|c|c|c|c|c|}
\hline \multicolumn{4}{|c|}{ Implicación de la venta directa en la vida familiar } & \multicolumn{2}{c|}{ Capacidad de ahorro } \\
\hline & Positivamente & Neutralmente & Negativamente & No & Sí \\
\hline Bogotá & $80,6 \%$ & $9,7 \%$ & $9,7 \%$ & $27,4 \%$ & $72,6 \%$ \\
\hline Lima & $93,9 \%$ & $6,1 \%$ & $0,0 \%$ & $20,4 \%$ & $79,6 \%$ \\
\hline Total & $84,4 \%$ & $8,7 \%$ & $6,9 \%$ & $25,4 \%$ & $74,6 \%$ \\
\hline
\end{tabular}


Los principales propósitos de vida son similares para las personas de Bogotá y de Lima. Esos propósitos son: mejorar económicamente (61\%), contar con estabilidad laboral $(18,5 \%)$ y estudiar $(17,9 \%)$. Sus principales expectativas de vida en la vejez son la estabilidad económica $(51,4 \%)$ y contar con buena salud y vida en familia $(41,6 \%)$ como se muestra en la Tabla 20.

Tabla 20: Principal propósito de su vida y expectativa de la vejez

\begin{tabular}{|l|c|c|c|c|c|c|c|c|}
\hline \multicolumn{4}{|c|}{ Principal propósito de su vida } & \multicolumn{5}{c|}{ Expectativa de la vejez } \\
\hline & $\begin{array}{c}\text { Mejorar lo } \\
\text { económico }\end{array}$ & $\begin{array}{c}\text { Estabilidad } \\
\text { familiar }\end{array}$ & Estudiar & $\begin{array}{c}\text { Ayudar a } \\
\text { otros }\end{array}$ & $\begin{array}{c}\text { Estabilidad } \\
\text { económica }\end{array}$ & $\begin{array}{c}\text { Con salud } \\
\text { y en familia }\end{array}$ & Indiferente & Trabajando \\
\hline Bogotá & $58,1 \%$ & $20,2 \%$ & $20,2 \%$ & $1,6 \%$ & $54,8 \%$ & $42,7 \%$ & $0,8 \%$ & $1,6 \%$ \\
\hline Lima & $69,4 \%$ & $14,3 \%$ & $12,2 \%$ & $4,1 \%$ & $42,8 \%$ & $38,7 \%$ & $12,2 \%$ & $6,1 \%$ \\
\hline Total & $61,3 \%$ & $18,5 \%$ & $17,9 \%$ & $2,3 \%$ & $51,4 \%$ & $41,6 \%$ & $4,0 \%$ & $2,9 \%$ \\
\hline
\end{tabular}

Los principales logros en la vida laboral para el conjunto de las personas participantes del estudio son la obtención de aprendizajes $(26,6 \%)$, mejoras económicas $(24,2 \%)$, éxito laboral $(21,4 \%)$ e independencia laboral $(13,9 \%)$. Según estos datos, en general, no hay un logro porcentualmente significativo con relación a los demás, sin embargo, por ciudad, se destaca el logro económico para las personas de Lima (40,9\%), como se muestra en la Tabla 21.

Tabla 21: Logros más importantes en su vida laboral a la fecha de la entrevista

\begin{tabular}{|l|c|c|c|c|c|c|}
\hline & $\begin{array}{c}\text { Obtener } \\
\text { aprendizajes }\end{array}$ & $\begin{array}{c}\text { Lograr } \\
\text { independencia }\end{array}$ & $\begin{array}{c}\text { Tener éxito } \\
\text { laboral }\end{array}$ & $\begin{array}{c}\text { Obtener } \\
\text { satisfacción } \\
\text { personal }\end{array}$ & $\begin{array}{c}\text { Alcanzar } \\
\text { logros } \\
\text { económicos }\end{array}$ & $\begin{array}{c}\text { Ninguno } \\
\text { logro }\end{array}$ \\
\hline Bogotá & $29,0 \%$ & $16,1 \%$ & $24,2 \%$ & $7,3 \%$ & $18,8 \%$ & $5,6 \%$ \\
\hline Lima & $20,4 \%$ & $8,2 \%$ & $14,3 \%$ & $12,2 \%$ & $40,9 \%$ & $4,1 \%$ \\
\hline Total & $26,6 \%$ & $13,9 \%$ & $21,4 \%$ & $8,7 \%$ & $24,2 \%$ & $5,2 \%$ \\
\hline
\end{tabular}

\section{Análisis multivariado}

Usando el Test de Fisher para las variables cualitativas (sexo, vivienda propia, estado civil y capacidad de ahorro) con una confiabilidad del $95 \%$, los datos de la Tabla 22 indican que no hay evidencia suficiente para afirmar que la proporción de las muestras de encuestados de Bogotá y de Lima son diferentes. Es decir que, cuando se analizan los datos de los participantes del estudio para estas variables, no es necesario desagregar el análisis de las muestras para cada una de las dos ciudades y se puede hacer para el conjunto de la población, indicando con esto que la venta directa es una oportunidad laboral que no discrimina las personas según las variables antes mencionadas como lo señala Sousa (2013).

Tabla 22: P-valor para el Test de Fisher para la diferencia proporcional entre las personas de Bogotá y Lima en cuanto a sexo, posesión de vivienda, esta civil y capacidad de ahorro

\begin{tabular}{|c|c|c|c|c|}
\hline Variable & Sexo & Vivienda propia & Estado civil & Capacidad de ahorro \\
\hline P-valor & 0.887 & 0.066 & 0.1016 & 0.4389 \\
\hline
\end{tabular}

También, utilizando el test de Fischer, con confiabilidad del $95 \%$, los datos de la prueba de la Tabla 23 indican que las muestras de las dos ciudades son similares en cuanto a las variables "Logro más importante en la vida laboral", "Implicación de la venta directa en la vida familiar" y "Razón de laborar en venta directa" excluyendo, respectivamente, las respuestas "Éxito laboral", "Implicación negativa de la venta" y "Beneficios de los productos". Estos datos evidencian que hay diferencia en el resultado cuando los datos se analizan de forma agregada que cuando se analizan de forma desagregada, como lo afirman Banbura y Modugno (2014).

Tabla 23: P-valor para el Test de Fisher para independencia entre las personas de Bogotá y Lima en cuanto a logro más importante, incidencia del multinivel en la vida familiar y razón de laborar en esa actividad

\begin{tabular}{|l|c|c|c|}
\hline Variable & $\begin{array}{c}\text { Logro más importante en la } \\
\text { vida laboral }\end{array}$ & $\begin{array}{c}\text { Implicación del multinivel en la } \\
\text { vida familiar }\end{array}$ & $\begin{array}{c}\text { Razón de laborar en venta } \\
\text { directa }\end{array}$ \\
\hline P-valor agregado & 0.0144 & 0.0379 & 0.036 \\
\hline P-valor desagregado & 0.06 & 0.5564 & 0.3631 \\
\hline
\end{tabular}


Igualmente, usando el test de Fisher, la distribución F de Fisher y la distribución T de Student, con confiabilidad del $95 \%$, para el análisis de la edad, la escolaridad, la satisfacción en venta directa y calidad de vida, los datos de la Tabla 24 indican que no hay evidencia para considerar la dependencia de cada una de estas variables respecto a la ciudad en que se realizó la encuesta, es decir, que cuando se discrimina por ciudad no hay suficiente evidencia para asegurar que las medias de las variables mencionadas son distintas de una ciudad con respecto de la otra.

Tabla 24: Independencia y diferencia de medias para la edad, la escolaridad, la calidad de vida y la satisfacción de la venta multinivel para las personas de Bogotá y Lima

\begin{tabular}{|l|c|c|c|c|}
\hline Variable & Edad & $\begin{array}{c}\text { Escolaridad (ordenada } \\
\text { por grado académico) }\end{array}$ & Calidad de vida & $\begin{array}{c}\text { Satisfacción en } \\
\text { venta directa }\end{array}$ \\
\hline P-valor test de independencia & 0.089 & 0.271 & 0.214 & 0.209 \\
\hline P-valor cociente de varianzas & 0.081 & 0.372 & 0.041 & 0.3720 \\
\hline P-valor diferencia de medias & 0.0733 & 0.1224 & 0.4667 & 0.0473 \\
\hline
\end{tabular}

Mediante el análisis de varianza, con nivel de confianza del $95 \%$, se puede concluir que las personas que laboran en venta directa por el interés de servicio y el beneficio de los productos son personas con edad promedio de 44 años, mientras que las que laboran en esta actividad por beneficios económicos y flexibilidad en el tiempo, su edad promedio es de 36 años, y la media de las que laboran porque simplemente se presentó la oportunidad es de 37 años, como lo muestra la Tabla 25.

También en la Tabla 25, mediante análisis de varianza con $95 \%$ de confianza, los datos indican que las personas con más hijos laboran en venta directa por el beneficio de los productos y el interés de servicio, mientras que las que tienen menor número de hijos lo hacen por beneficios económicos y flexibilidad del tiempo. Los que afirman laborar porque se presentó la oportunidad tienen, en promedio, 1,6 hijos. Estos resultados hacen inferir que el número de hijos puede ser una variable condicionante para la realización de esta labor, la cual no es tan accesible a todo interesado como lo afirma Sousa (2013).

Tabla 25: Edad y número0 de hijos versus razón de laborar en venta directa

\begin{tabular}{|l|l|c|c|}
\hline \multirow{2}{*}{$\begin{array}{l}\text { Grupos sin diferencias } \\
\text { significativas en la media }\end{array}$} & Razón de laborar en venta directa & Edad promedio & $\begin{array}{l}\text { Promedio de } \\
\text { número de hijos }\end{array}$ \\
\hline \multirow{2}{*}{ Grupo A } & Interés de servicio & 45,10 & 1,89 \\
\cline { 2 - 4 } & Beneficio de los productos & 44,15 & 1,92 \\
\hline Grupo B & Se presentó la oportunidad & 37,45 & 1,63 \\
\hline \multirow{2}{*}{ Grupo C } & Beneficios económicos & 36,99 & 1,16 \\
\cline { 2 - 4 } & Flexibilidad del tiempo & 36,69 & 0,92 \\
\hline
\end{tabular}

El análisis de varianza, con $95 \%$ de confianza, indica que no hay evidencia estadística para afirmar que el nivel de formación académica de las personas que están en venta directa, ni el poseer o no vivienda propia, o mantener relación positiva o no en la vida familiar, son criterios para explicar las razones por las que las personas optaron por esta actividad. Según el test de Fisher, tampoco se puede afirmar que haya diferencia entre la percepción de los hombres y la de las mujeres en cuanto a la satisfacción por su actividad en venta directa. Tampoco se puede evidenciar que haya diferencia estadística en la satisfacción en venta directa entre las personas de diferente estado civil y entre personas con diferentes expectativas de su vejez.

Igualmente, el análisis de varianza indica que las personas que manifestaron haber ocupado más empleos en su historia laboral de diez años, tienen significativamente, mayores promedios de salario que las personas con menos empleos; no hay evidencia para afirmar que la cantidad de horas trabajadas por las personas que tuvieron un solo empleo es significativamente distinta a las que tuvieron dos, tres o más empleos; no hay diferencia en el nivel de cargo desempeñado según el número de empleos, el tipo de contrato de vinculación laboral ni la percepción de la relación con los jefes; el promedio del salario, el número de horas diarias trabajadas, el tipo de contrato, el nivel del cargo desempeñado por las personas o el tipo de relaciones con los jefes no son determinantes de la razón para realizar la venta directa. Des estos datos se puede inferir que la movilidad laboral en esta actividad se presenta por la búsqueda de mejoras económicas más que por la cantidad de horas laboradas, como lo afirman Correa y Orjuela (2007). 


\section{Componentes principales}

Las variables edad, número de hijos, período de tiempo sin empleo en los últimos diez años y tiempo de laborar en venta directa, junto con las variables escolaridad, satisfacción en venta directa, calidad de vida e incidencia de la venta directa en las relaciones familiares, se analizan en conjunto con la técnica "Análisis de Componentes Principales (PCA)", donde, a partir de la matriz de correlación, se explica, en menores dimensiones, una porción de la varianza de los datos (Kassambara, 2017). La prueba estadística utilizada para la identificación de los componentes principales es correspondiente a la ecuación (1).

$$
z_{i j}=\phi_{1 j} x_{i 1}+\phi_{2 j} x_{i 2}+\cdots+\phi_{8 j} x_{i 8}
$$

Donde: $1 \leq i \leq 174 ; Z j$ con $1 \leq j \leq 8 ; \phi_{k j}$ con $1 \leq k \leq 8 ; y Z_{j}$ se calculó, en términos de los valores propios $\lambda_{j}$ de la matriz de correlaciones asociada a las ocho variables, y para esto se utilizó la ecuación (2)

$$
\frac{\lambda_{j}}{\sum_{i=1}^{8} \operatorname{Var}\left(Z_{i}\right)}=\frac{\lambda_{j}}{\sum_{i=1}^{8} \lambda_{i}}
$$

Donde $\operatorname{Var}\left(Z_{j}\right)$ es la varianza total presente en la componente j.

La porción de varianza explicada por las direcciones de las componentes principales $\phi_{k j}$ con $1 \leq j \leq 8$ se representa en la Figura 1 donde se evidencia que el $54,7 \%$ de la variabilidad está contenido en las tres primeras componentes principales $(26,2 \%+15,5 \%+13 \%)$, es decir, si se reducen los datos a tan sólo tres dimensiones se pueden analizar sus relaciones en cuanto a variabilidad en más del $50 \%$.

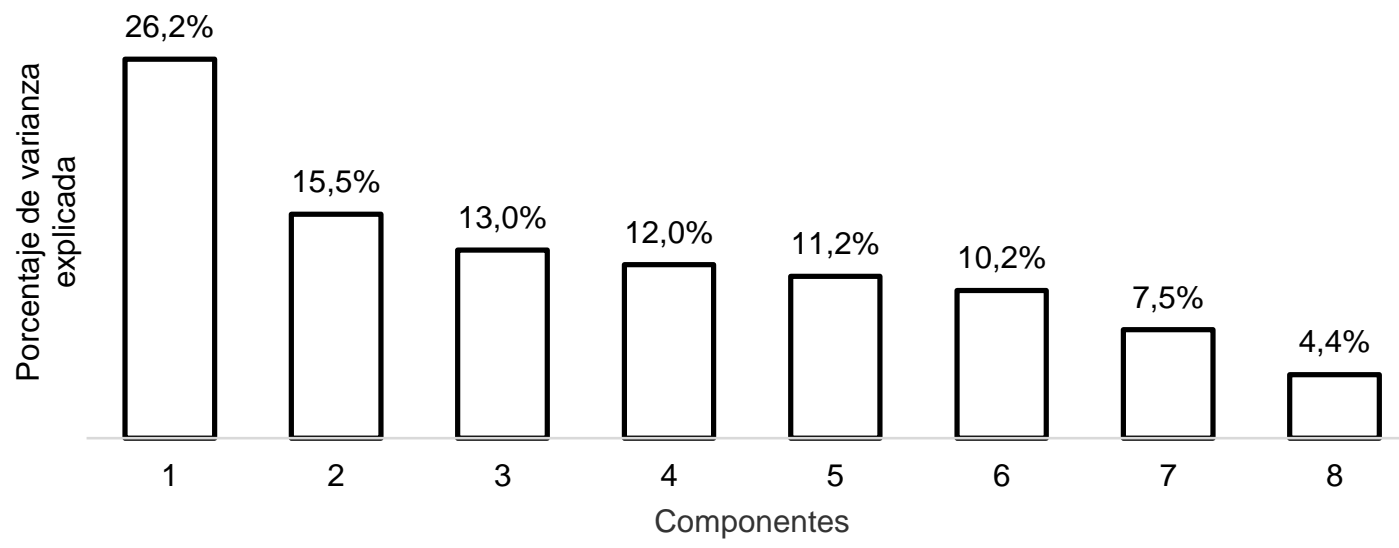

Fig. 1: Porcentaje de varianza explicada por cada componente principal

El porcentaje de contribución de varianza en cada una de las tres primeras componentes se desagrega por variables en la Tabla 26. La componente 1, que explica el $26,2 \%$ de la variación de los rasgos de las personas dedicadas a venta multinivel, está conformada principalmente por la edad, el número de hijos y el tiempo de labor en venta directa. Las componentes 2 y 3 que explican el $15,5 \%$ y el $13 \%$, respectivamente, de la variación de los rasgos, destacan la "calidad de vida" mayormente valorada como buena $(65,9 \%$, Tabla 19) y la "satisfacción de laborar en venta directa", mayormente valorada como satisfactoria (64,2 \%, Tabla 15); ambas están relacionadas con su autopercepción como los dos rasgos descriptores más relevantes de la muestra de la población encuestada.

Tabla 26: Porcentaje de contribución de la varianza de las primeras tres componentes

\begin{tabular}{|l|c|l|c|l|c|}
\hline \multicolumn{2}{|c|}{ Componente 1 } & \multicolumn{2}{c|}{ Componente 2 Componente 3 } & \multicolumn{2}{c|}{} \\
\hline Edad & $33,1 \%$ & Calidad de vida & $50,1 \%$ & $\begin{array}{l}\text { Satisfacción en } \\
\text { multinivel }\end{array}$ & $57,2 \%$ \\
\hline Hijos & $31,4 \%$ & $\begin{array}{l}\text { Tiempo mayor de } \\
\text { desempleo }\end{array}$ & $24,9 \%$ & $\begin{array}{l}\text { Incidencia relaciones } \\
\text { familiares }\end{array}$ & $31,0 \%$ \\
\hline Tiempo en multinivel & $27,6 \%$ & Escolaridad & $14,7 \%$ & Escolaridad & $10,7 \%$ \\
\hline Escolaridad & $10,1 \%$ & $\begin{array}{l}\text { Satisfacción en venta } \\
\text { directa }\end{array}$ & $4,1 \%$ & Edad & $1,8 \%$ \\
\hline $\begin{array}{l}\text { Incidencia relaciones } \\
\text { familiares }\end{array}$ & $4.0 \%$ & Edad & $3,9 \%$ & Calidad de vida & $0,4 \%$ \\
\hline
\end{tabular}




\section{CONCLUSIONES}

Analizar los rasgos y las trayectorias laborales de las personas en un contexto de transformaciones del entorno económico y laboral como el actual, en ciudades como Bogotá y Lima, permite identificar los diferentes cambios que caracterizan el mundo del trabajo y la manera como estos cambios afectan a las personas en particular y a la sociedad en general. La evidencia muestra que las trayectorias laborales de las personas en las últimas tres décadas se han reconfigurado al pasar de ser trayectorias que se daban en una sola organización mediante el desarrollo de destrezas en un determinado campo del trabajo, mediadas por contratos de carácter indefinido basado en la confianza, a trayectorias laborales que se desarrollan en varias organizaciones, con contratos a término fijo, con múltiples destrezas evaluadas en términos de beneficios directos e inmediatos.

Estudiar la trayectoria laboral de las personas que trabajan en la modalidad de venta multinivel ayuda a comprender tanto su contexto sociolaboral como las formas en que enfrentan el mercado laboral y cómo se perciben a sí mismas. Los resultados del estudio son un insumo para generar reflexión en relación con las circunstancias actuales del trabajo que vivencian las personas como actores involucrados en este tipo de actividad.

\section{AGRADECIMIENTOS}

Los autores agradecen a la Dra. María Claudia Peralta, quien fue la persona que sugirió el tema de la investigación y por haber colaborado de forma incondicional el todo el proceso de la investigación, en particular con el diseño y validación del cuestionario de la encuesta que se aplicó para la recogida de la información de los participantes del estudio y también por sus observaciones en la redacción del este artículo. También se agradece a las directivas del área de investigación de la Universidad Alas Peruanas por el apoyo para el desarrollo de esta Investigación.

\section{REFERENCIAS}

Benería, L., Trabajo productivo/reproductivo, pobreza y políticas de conciliación. Nómadas, (24), 8-21 (2006).

Bernal-Torres, C.A., y otros tres autores, Informal Workers in the Streets: An Integral View. DOI: 10.2478/mjss-2018-0156, Mediterranean Journal of Social Sciences, 9(5), 251,260 (2018).

Blanch J., Teoría de las relaciones laborales. Tomo I: Fundamentos. Tomo II: Desafíos, ISBN: 84-8318-301-3 EDIUOC Barcelona, Espáña (2003).

Blanco-Valbuena, C.E. y tres autores más, Industrias Creativas y Culturales: Estudio desde el enfoque de la Gestión del Conocimiento. http://dx.doi.org/10.4067/S0718-07642018000300015, Información tecnológica, 29(3), 15-28 (2018)

Cárdenas, D. y Fuchs-Tarlovsky, V., Is multi-level marketing of nutrition supplements a legal and an ethical practice? https://doi.org/10.1016/j.clnesp.2018.03.118, Clinical nutrition ESPEN, 25, 133-138 (2018).

Castillo, V., Rojo, S. Novik, M., Trayectorias laborales y rotación del empleo. Restricciones para el desarrollo de competencias técnicas. Asociación argentina de especialistas en estudios de trabajo, Buenos Aires. Argentina (2005).

Cepal - IOT., Coyuntura laboral en América Latina y el Caribe: La inserción laboral de las personas mayores: necesidades y opciones. Cepal y OIT, Santiago de Chile, Chile (2018).

De Cuyper N., Notelaers, G. y De Witte, H., Transitioning between temporary and permanent employment: a two-wave study on the entrapment, the stepping stone and the selection, https://doi.org/10.1348/096317908X299755, Journal of Occupational and Organizational Psychology 82, 67-88 (2009).

González, M.M., Las empresas multinivel como modelo distópico: estudio de representaciones sociales. Aposta. Revista de Ciencias Sociales, (62), 1-27 (2014).

Grote, G. y Raeder, S., Careers and identity in flexible working: Do flexible identities fare better? DOI: 10.1177/0018726708100358, Human Relations, 62(2), 219-244 (2009).

Guadarrama, R., Hualde, A. y López, S., Precariedad laboral, heterogeneidad ocupacional y desigualdades de género en México en el contexto de la crisis actual: una propuesta 188 metodológica", documento de trabajo del grupo de trabajo: "Heterogeneidad ocupacional, precariedad laboral y desigualdades de género en México". Revista Mexicana de Sociología, 74(2), 213-243 (2012).

Kassambara, A., Parctical guide to Principal Component methods in R. Create Space Independent Publishing Platform; 1 edition. ISBN-10: 1975721136 y ISBN-13: 978-1975721138. El autor: Edición virtual (2017).

Kim, B.J. y otros tres autores. Network marketing on a small-world network. Physica A: Statistical Mechanics and its Applications, 360(2), 493-504 (2006).

Laczniak, G. y Murpdy, P., The role of normative marketing ethics. https://doi.org/10.1016/j.jbusres.2018.07.036, Journal of Business Research, 95, 401-407 (2019). 
Legara, E., y otros cuatro autores, Earning potential in multilevel marketing enterprises. https://doi.org/10.1016/j.physa.2008.04.009, Physica A: Statistical Mechanics and its Applications, 387(19- 20), 48894895 (2008).

López-Andreu, M., y Verd, J., Employment instability and economic crisis in Spain: What are the elements that make a difference in the trajectories of younger adults? DOI: 10.1080/14616696.2016.1207791, European Societies, 18(4), 315335 (2016).

Merlin, M., A Study on Direct Selling Through Multi-Level Marketing. International Journal of Advancements in Research \& Technology, 1(4), 1- 3 (2012).

Muncy, J., Ethical issues in multilevel marketing: is it a legitimate business or just another pyramid scheme? https://doi.org/10.1080/10528008.2004.11488877, Marketing Education Review, 14(3) (2012).

Muñiz Terra, L., Carreras y trayectorias laborales: una revisión crítica de las principales aproximaciones teóricometodológicas para su abordaje. Revista latinoamericana de metodología de las Ciencias Sociales, 2(1), 36-65 (2012).

Nam, T., Technology usage expected job sustainability, and perceived job insecurity. https://doi.org/10.1016/j.techfore.2018.08.017, Technological Forecasting and Social Change, 138, 155-165 (2019).

Olaniyan, O. S., y. Hystad, S.W., Employees' psychological capital, job satisfaction, insecurity, and intentions to quit: The direct and indirect effects of authentic leadership. https://doi.org/10.1016/j.rpto.2016.09.003, Revista de Psicología del Trabajo y de las Organizaciones, 32(3), 163-171 (2016).

Orejuela-Gómez, J.J., y Correa-García, A., Trayectorias laborales y relacionales: una nueva estética. https://doi.org/10.21500/22563202.500, Revista Científica Guillermo de Ockham. 5(1). 59 - 72 (2007).

Peralta- Gómez, M.C., Significados, cambios y contexto actual de trabajo.: Estudio interpretativo. Diversitas: perspectivas en psicología, 8(1), 165-182 (2012).

Roberti, M.E., Rupturas y subjetividades: Un acercamiento a la perspectiva de las Trayectorias Laborales. Trabajo y Sociedad (2012).

Schiffauer, L., Let's get rich: Multilevel marketing and the moral economy in Siberia. DOI: 10.1177/0308275X18775207, Critique of Anthropology, 38(3) 285-302 (2018).

Soto, A., Narrativas de Profesionales Chilenos sobre sus Trayectorias Laborales: La Construcción de Identidades en el Trabajo. http://dx.doi.org/10.4067/S0718-22282011000100002, Revista PSYKHE, Pontificia Universidad Católica de Chile, 20(1), 15-27 (2012).

Sousa, I., Marketing multinivel en el Siglo XXI. Universidad Politécnica de Cartagena, España (2013).

Vandernat, P. y Keed, W., Multilevel Marketing: A Historical Perspective. Journal of Public Policy and Marketing, 37(7), 345-359 (2013).

Verd, J. M., Barranco, O. y Bolíbar, M., Youth unemployment and employment trajectories in Spain during the Great Recession: what are the determinants? Journal for Labour Market Research, 53(1), 4 (2019).

World Federation of Direct Selling Associations., Annual Report. Disponible en: https://wfdsa.org/initiatives-andresources/publications/ (2018). 